\title{
Implicit force control approach for safe physical robot-to-human object handover
}

\author{
Paramin Neranon \\ Department of Mechanical Engineering, Faculty of Engineering, Prince of Songkla University, Thailand
}

\begin{tabular}{l}
\hline \hline Article Info \\
\hline Article history: \\
Received Jan 10, 2019 \\
Revised May 1, 2019 \\
Accepted Jul 1, 2019 \\
\hline
\end{tabular}

\section{Keywords:}

Fuzzy logic control Human-robot interaction Implicit force control Proportional integral control System dynamic model

\begin{abstract}
This research focuses on the development of the conceptual frameworks of human-human interaction applied for a robotic behaviour-based approach for safe physical human-robot interaction. The control has been constructed based on understanding the dynamic and kinematic behavioural characteristics of how two humans pass an object to each other. This has enabled a KR-16-KUKA robot to naturally interact with a human so as to facilitate the dexterous transfer of an object in an effective manner. Implicit force control based on Proportional Integral and Fuzzy Logic Control which allows the robot end effector's trajectory to be moderated based on the applied force in real-time was adopted. The experimental results have confirmed that the quantitative performance of the force-controlled robot is close to that of the human and can be considered acceptable for human-robot interaction. Furthermore, the control based Fuzzy Logic Control was shown to be slightly superior performance compared to Proportional Integral control.
\end{abstract}

Copyright $@ 2020$ Institute of Advanced Engineering and Science. All rights reserved.

\section{Corresponding Author:}

Paramin Neranon,

Department of Mechanical Engineering,

Prince of Songkla University,

Hat Yai, Songkhla, Thailand, 90110.

Email: paramin.n@psu.ac.th

\section{INTRODUCTION}

Robots are poised to fill a growing number of roles in today's society. These include various applications in automated factories, medical, service and other facilities since they continue to be successfully used to improve productivity, quality, accuracy, and reliability. In the next generation of intelligent robots, it will be expected to have a larger operating range and to offer increased flexibility, as well as sharing the workspace with humans in order to further improve productivity. To achieve successful collaboration, robots are required to collaborate with humans in a safe and effective manner. However, in Human-Robot Interaction (HRI) context, robots themselves have to appropriately cope with many complicated uncertainties introduced by the dynamic nature of the human environment. Thus, it is very crucial to necessitate the very careful design of a robot control strategy and its implementation in order to protect the human from the risk of harm or injury by the robot [1-2].

Many different types of HRI have been proposed, and one of the common HRI scenarios is object handover, with robots used as household aids or assistive devices for the elderly or patients. For an instant, robots will become trustworthy helpers of humans and can perform a variety of services at homes such as safely and effortlessly passing common objects of daily life without dropping [3]. Cakmak and his colleagues [4] studied Human-Human Interaction (HHI), which are an essential aspect of HRI object handover applications involving an assistive robot. The most significant human preferences whilst performing an object handover task were also identified and evaluated. Parastegari and his researchers [5] delivered the human modelling in $\mathrm{HHI}$ object handover which can predict the object transferring point and its 
trajectory before implementing to physical HRI object handover. Some researchers [6-7] conducted the observation of HHI object handover before implementing the handover behavioural control strategy on HRI. Handlers and receivers were evaluated in terms of gestures when approaching, carrying, reaching and transferring.

Other researchers, who also investigated different issues to improve HRI object handover interactive tasks, have studied how a human applies grip force during an object transfer task [8-9].

Most of the challenges of the development of trustworthy HRI are safety, predictability and usefulness since it is further complicated by the dynamic nature of the human environment. Thus, it necessitates very careful design and implementation of the robot behavioural control strategy in order to significantly improve the success rate of object handover tasks and also protect the human cooperator from the risk of harm or injury by the robot [10]. Reed and his colleagues [11] stated that having examined the principle of human haptic interaction when two humans work together in a joint effort to complete a shared task is crucial in designing an effective HRI system. As an extensive review, this body of work, therefore, emphasizes on developing a robot behavioural control strategy which allows a robot manipulator to be configured with the capability of generating natural and synchronized responses with a human partner to facilitate safe and effective collaboration while performing HRI object handover tasks. To achieve this, the behavioural and dynamic characteristics of both handler and receiver humans while performing object handover tasks were initially investigated before being implemented on a robot to mimic the same interactive tasks with a human partner. Finally, the robot's performance was then compared with the outcomes of the HHI tests in order to assess the robot behaviour-based control system.

\section{CONCEPTUAL DESIGN, TEST PROCEDURE AND EVALUATION OF HUMAN-TO- HUMAN OBJECT HANDOVER}

As mentioned, it is crucial to understand the kinematics and dynamics of HHI object handover behaviour in order to design and develop an appropriate set of control strategies for robust, behaviour-based, HRI. Thus, to mimic a real-world object handover task, a set of HHI tests has been undertaken to investigate how the handler and receiver behave whilst performing object handover in a real shared workspace, similar to passing the baton in a relay race. The goals of the handover experiments are to evaluate human physical characteristics in HHI in terms of a) How the handler and receiver regulate their interactive forces whilst executing the interactive tasks under different velocities and masses conditions, b) How the handler releases the object to be transferred in a natural and timely manner, c) How long the object handover process takes, and d) How much work is done by the participant pair. The provided dynamic and kinematic information of the $\mathrm{HHI}$ characteristics is subsequently used in the robot behaviour-based control strategies.

The workspace of the object handover task can be divided into three distinct phases i.e. sending, transfer and receiving. A baton-type object to be transferred was designed to facilitate the accomplishment of the characterization of the haptic human dynamic interaction. The handling interactive forces in $\mathrm{x}-\mathrm{y}-\mathrm{z}$ axis (fx, fy and fz) applied by the giver and receiver were measured by an ATI mini40 F/T sensor and collected in real-time every $4 \mathrm{~ms}(250 \mathrm{~Hz})$. The object velocity can be estimated by integrating an output signal from a DE-ACCM accelerometer sensor. Eighteen participants from Prince of Songkla University were randomly selected and employed in the handover tasks under different conditions, i.e. various transfer velocities of 10 , 50 and $100 \mathrm{~mm} / \mathrm{s}$ and masses added with the load capacity of $0.2,0.6$ and $1.0 \mathrm{~kg}$. A detailed description is clearly given in the research paper by Neranon [12]. Test evaluation of the interactive experiments provides an understanding of the physical HHI dynamic responses in the object handover tasks. Additionally, various key features were used to be investigated before being implemented in the development of human-like robot control strategies able to effectively perform human-like functions in HRI object handover. These consist of handling interactive force (fint) profiles against time ( $\mathrm{t}$ ), the maximum interactive force (fmax), the transfer time (Ttrf), and work done (W). The processes of graph normalization and curve fitting were applied due to the elimination of undesirable variations from the achieved interactive force data. The overall experimental results of the $\mathrm{HHI}$ object handover are summarised in Table 1.

\section{CONCEPTUAL FRAMEWORK FOR ROBOT-TO-HUMAN OBJECT HANDOVER INTERACTION}

Conceptual design of HRI attempts to maximize the benefits of collaboration between a human and a robot to successfully accomplish an effective object handover task. As detailed in the last section, the haptic dynamic responses between the giver and receiver participants in the handover tasks were initially investigated, and this offers a better understanding of the dynamic and kinematic HHI. These findings were potentially used as the conceptual framework for the robot-to-human object handover task. Behaviour-based 
robotics control enables the robot to transfer the baton-type device to the human receiver in a safe, timely and natural manner.

Table 1. Comparisons of the Required Features in HHI Object Handover Tasks under the Various Velocity and Mass Conditions

\begin{tabular}{ccccccc}
\hline \multicolumn{7}{c}{ Transfer time (s) } \\
\hline Velocity (mm/s) & Mass 0.42kg & SD (s) & Mass 0.82kg & SD (s) & Mass 1.22kg & SD (s) \\
10 & 0.54 & 0.05 & 0.52 & 0.08 & 0.48 & 0.08 \\
50 & 0.44 & 0.04 & 0.43 & 0.04 & 0.38 & 0.04 \\
100 & 0.37 & 0.03 & 0.35 & 0.04 & 0.30 & 0.03 \\
& \multicolumn{7}{c}{ Force max (N) } \\
Velocity (mm/s) & Mass 0.42kg & SD (N) & Mass 0.82kg & SD (N) & Mass 1.22kg & SD (N) \\
10 & 0.85 & 0.19 & 1.46 & 0.32 & 2.26 & 0.56 \\
50 & 1.32 & 0.28 & 2.44 & 0.41 & 3.32 & 0.41 \\
100 & 2.43 & 0.46 & 2.79 & 0.58 & 3.63 & 0.45 \\
& & Work done (mJ or N.mm) & & SD (mJ) \\
Velocity (mm/s) & Mass 0.42kg & SD (mJ) & Mass 0.82kg & SD (mJ) & Mass 1.22kg & SD \\
10 & 1.9 & 0.3 & 2.9 & 0.3 & 4.1 & 0.8 \\
50 & 11.0 & 1.6 & 21.8 & 2.5 & 25.8 & 3.2 \\
100 & 34.3 & 6.6 & 38.6 & 7.5 & 42.4 & 6.7 \\
\hline
\end{tabular}

\subsection{A Simplest Dynamic Mechanical Model of the HRI Object Handover System}

This section describes the simplest dynamic model of the robot-force control system in HRI object handover. The control scheme was designed based on the study of active compliant motion control methods for rigid manipulators suggested by Schutter [13]. The system is initially assumed to be an equivalent lumped-mass system, which is illustrated in Figure 1. The KUKA KR-16 robot is represented by the rigid body model (mr) associated with an effective viscous damping (br) to ground, and the robot itself can generate driving force of F. The ATI force/torque sensor is modelled as a spring and dashpot system and mounted between the robot and the robot gripper, in which the stiffness and viscous damping are ks and bs respectively. The robot gripper has been added to the model, with its stiffness, viscous damping and mass represented as $\mathrm{kg}$, bg and $\mathrm{mg}$ respectively. The human model is confined to that component of the environment which is contacted by the robot end effector. The rigid body mass of the human while holding the rigid baton-type object represents as mw, and his/her viscous damping and stiffness are bw and kw [14]. In addition, the displacements of the masses $(\mathrm{mr}, \mathrm{mg}$ and $\mathrm{mw}$ ) are respectively defined as $\mathrm{xr}, \mathrm{xg}$ and $\mathrm{xw}$. This system modelling parameters can be estimated using MATLAB system identification $[15,16]$

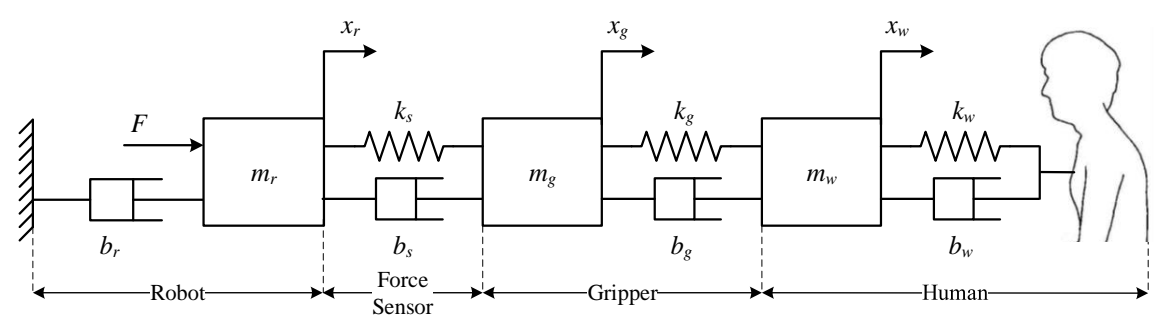

Figure 1. A dynamic mechanical model of the robot-to-human object handover

By applying Newton's second law, the dynamic equations of the rigid body masses (mr, mg and $\mathrm{mw}$ ) were calculated and subsequently used to design of the states-space representation for the robot dynamic model. It can be expressed as follows:

$$
\begin{aligned}
& m_{r} \ddot{x}_{r}(n)=F(n)-\left(b_{r} \dot{x}_{r}(n)\right)-k_{s}\left(x_{r}(n)-x_{g}(n)\right)-b_{s}\left(\dot{x}_{r}(n)-\dot{x}_{g}(n)\right) \\
& m_{g} \ddot{x}_{g}(t)=\left[k_{s}\left(x_{r}-x_{g}\right)\right]+\left[b_{s}\left(\dot{x}_{r}-\dot{x}_{g}\right)\right]-k_{g}\left(x_{g}-x_{w}\right)-b_{g}\left(\dot{x}_{g}-\dot{x}_{w}\right)
\end{aligned}
$$

and

$$
m_{w} \ddot{x}_{w}(t)=\left[k_{g}\left(x_{g}-x_{w}\right)\right]+\left[b_{g}\left(\dot{x}_{g}-\dot{x}_{w}\right)\right]-k_{w} x_{w}-b_{w} \dot{x}_{w}
$$


The design of the states space for the dynamic model of the robot force control system specifically requires the characteristic equations as defined below:

$$
\begin{aligned}
& \frac{d x_{r}(n)}{d t}=\dot{x}_{r}(n) \\
& \ddot{x}_{r}(n)=\frac{F(n)}{m_{r}}-\frac{\left(b_{r}+b_{s}\right) \dot{x}_{r}(n)}{m_{r}}+\frac{b_{s} \dot{x}_{g}(n)}{m_{r}}-\frac{k_{s} x_{r}(n)}{m_{r}}+\frac{k_{s} x_{g}(n)}{m_{r}} \\
& \frac{d x_{g}(n)}{d t}=\dot{x}_{g}(n) \\
& \ddot{x}_{g}(t)=\frac{b_{s} \dot{x}_{r}}{m_{g}}-\frac{\left(b_{s}+b_{g}\right) \dot{x}_{g}}{m_{g}}+\frac{b_{g} \dot{x}_{w}}{m_{g}}+\frac{k_{s} x_{r}}{m_{g}}-\frac{\left(k_{s}+k_{g}\right) x_{g}}{m_{g}}+\frac{k_{g} x_{w}}{m_{g}} \\
& \frac{d x_{w}(n)}{d t}=\dot{x}_{w}(n) \\
& \ddot{x}_{w}(t)=\frac{b_{g} \dot{x}_{g}}{m_{w}}-\frac{\left(b_{g}+b_{w}\right) \dot{x}_{w}}{m_{w}}+\frac{k_{g} x_{g}}{m_{w}}-\frac{\left(k_{g}+k_{w}\right) x_{w}}{m_{w}}
\end{aligned}
$$

In a vector-matrix form, the state space representation can be then written as:

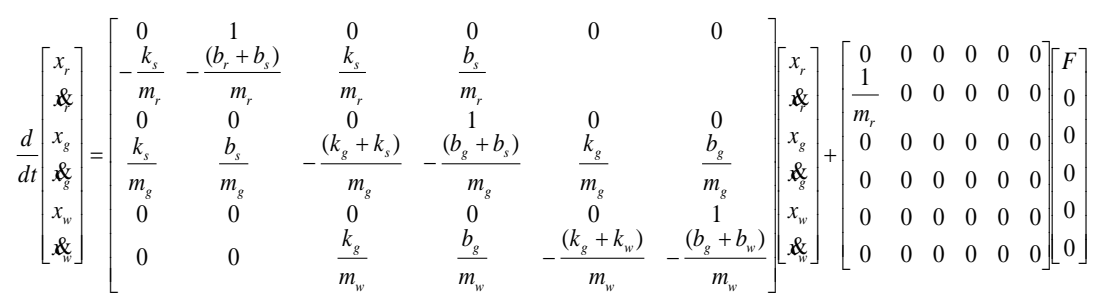

The system output is formulated as follows:

$$
y=\left[\begin{array}{llllll}
1 & 0 & 0 & 0 & 0 & 0 \\
0 & 0 & 1 & 0 & 0 & 0 \\
0 & 0 & 0 & 0 & 1 & 0
\end{array}\right]\left[\begin{array}{c}
x_{r} \\
\& \\
x_{g} \\
\& \\
x_{w} \\
\& \\
w
\end{array}\right]+[0] F .
$$

The system transfer functions are then computed and given by:

$$
G_{1}(s)=\frac{x_{r}(S)}{F(S)}=\frac{A}{D}, G_{2}(s)=\frac{x_{g}(S)}{F(S)}=\frac{B}{D} \text { and } G_{3}(s)=\frac{x_{w}(S)}{F(S)}=\frac{C}{D}
$$

where

$$
\begin{aligned}
& \mathrm{A}=\left(k_{g} k_{s}+k_{g} k_{w}+k_{s} k_{w}+b_{g} k_{s} S+b_{s} k_{g} S+b_{g} k_{w} S+b_{w} k_{g} S+b_{s} k_{w} S+b_{w} k_{s} S+b_{g} b_{s} S^{2}+\right. \\
& b_{g} b_{w} S^{2}+b_{s} b_{w} S^{2}+b_{g} m_{g} S^{3}+b_{g} m_{g} S^{3}+b_{g} m_{w} S^{3}+b_{w} m_{g} S^{3}+b_{s} m_{w} S^{3}+k_{g} m_{g} S^{2}+ \\
& \left.k_{g} m_{w} S^{2}+k_{w} m_{g} S^{2}+k_{s} m_{w} S^{2}+m_{g} m_{w} S^{4}\right) \\
& \mathrm{B}=\left(\left(k_{s}+S b_{s}\right)\left(k_{g}+k_{w}+b_{g} S+b_{w} S+m_{w} S^{2}\right)\right) \\
& \mathrm{C}=\left(\left(k_{g}+S b_{g}\right)\left(k_{s}+S b_{s}\right)\right) \\
& \mathrm{D}=\left(k_{g} k_{s} k_{w}+b_{g} b_{r} b_{s} S^{3}+b_{g} b_{r} b_{w} S^{3}+b_{g} b_{s} b_{w} S^{3}+b_{r} b_{s} b_{w} S^{3}+b_{g} b_{r} k_{s} S^{2}+b_{r} b_{s} k_{g} S^{2}+\right. \\
& b_{g} b_{r} k_{w} S^{2}+b_{r} b_{w} k_{g} S^{2}+b_{g} b_{s} k_{w} S^{2}+b_{g} b_{w} k_{s} S^{2}+b_{s} b_{w} k_{g} S^{2}+b_{r} b_{s} k_{w} S^{2}+b_{r} b_{w} k_{s} S^{2}+ \\
& b_{g} b_{r} m_{g} S^{4}+b_{g} b_{s} m_{g} S^{4}+b_{g} b_{s} m_{r} S^{4}+b_{g} b_{r} m_{w} S^{4}+b_{g} b_{w} m_{r} S^{4}+b_{r} b_{w} m_{g} S^{4}+b_{g} b_{s} m_{w} S^{4}+
\end{aligned}
$$




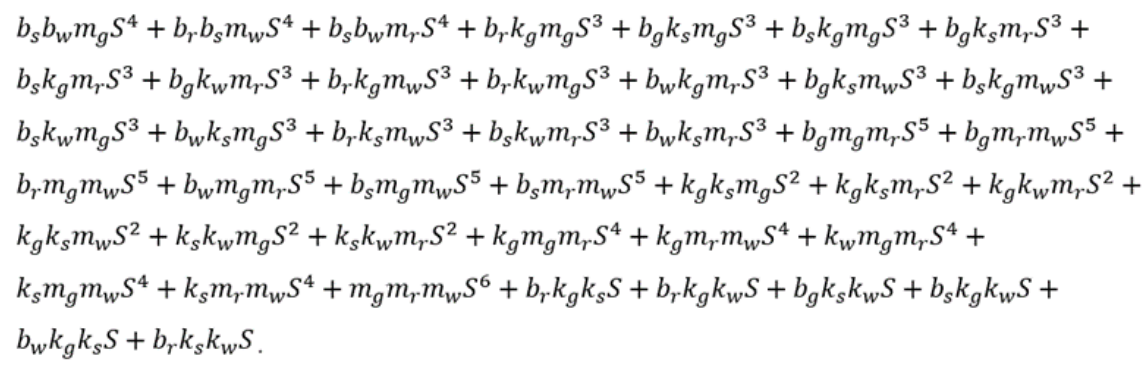

\subsection{Implementation of Implicit Force Control Approach for HRI Object Handover}

Robot implicit force control is a crucial requirement in the achievement of the robot's real-time path control in any physical interaction task. Practically, it is able to suitably control commercial robots using implicit force control because this type of robots has been designed as positioning devices. The control approach provides the force control rule which functions to respond to the human environment, track the desired forces, and compensate for variations in robot positioning at the contact surface. The advantages of using this force control technique are to avoid switching between position and force control loops, and both position and force control are handled in the same Cartesian direction. Hence, it delivers more reliability and stability of the HRI application [13,17]. Figure 2 illustrates a schematic of the implicit position-based force control applied for HRI object handover. Typically, there are two main control loops, in which the inner and outer loops refer to force control and position control respectively. For better understanding, consider the control block diagram. The $\mathrm{X}$ and $\mathrm{F}$ are $6 \times 1$ vectors representing the Cartesian position and orientation and Cartesian force and moment respectively. The $S$ is a $6 \times 6$ diagonal selection matrix, where each element becomes a one for position control or zero for no position control. Once the robot is in contact with a human, the input of the force control loop $(\Delta \mathrm{F})$ can be computed from the error between desired force (Fref) and actual force values (Fs) measured from a multi-axis force/torque sensor. An output of the force control (or a force control direction: $\mathrm{XOF}$ ) provides an equivalent position (X0), which is directly modified by an initial reference position $(\mathrm{X} O \mathrm{P})$. An incremental position $(\Delta \mathrm{X})$ is calculated by subtracting $\mathrm{X} 0$ from a current robot position $(\mathrm{X})$. Cartesian coordinates are conveyed by the position control and then transferred directly to the robot to modify its trajectory with an object environment position (XE).

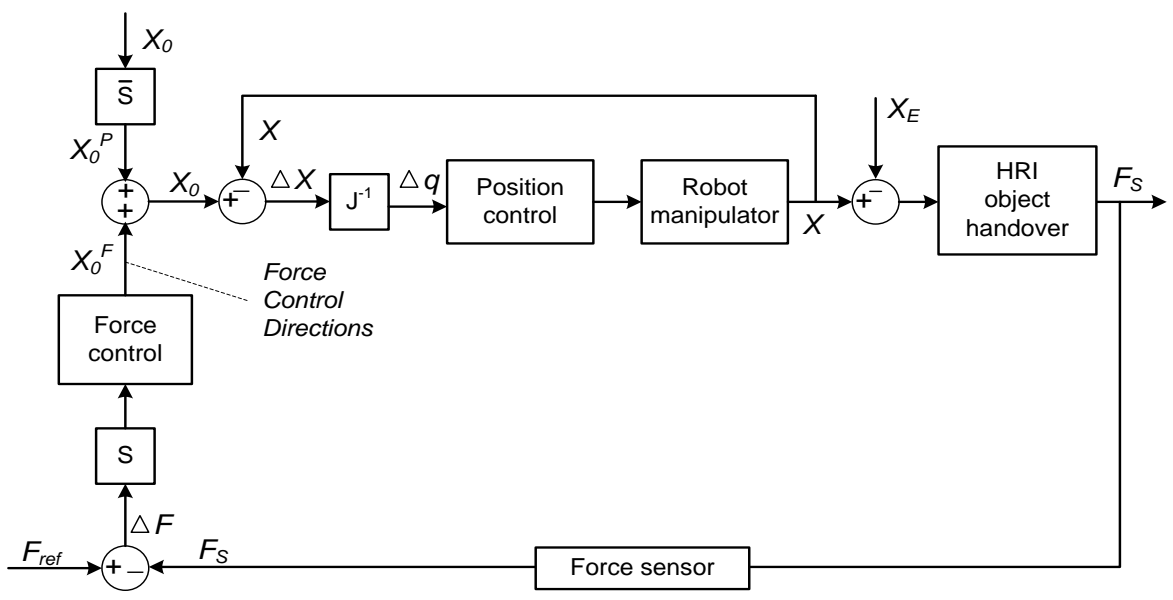

Figure 2. Position-based implicit force control

Figure 3 depicts an overall schematic diagram of the HRI system and how to specifically implement the implicit force control to the robot. In terms of hardware of the system, it consists of a 6-DOF KUKA KR16 robot arm, a KUKA-KRC2 ED05 robot controller and a manual control panel which are available in Robotic LAB at Prince of Songkla University. An ATI multi-axis force/torque and position sensors were utilized to detect the handling interactive force between the human and robot (via the robot's end effector) and object position respectively. The force control software and the host PC-robot controller communication via an Ethernet port using the TCP/IP protocol were successfully developed through Robot operating system (ROS) by updating every $4 \mathrm{~ms}$ or $250 \mathrm{~Hz}$. 


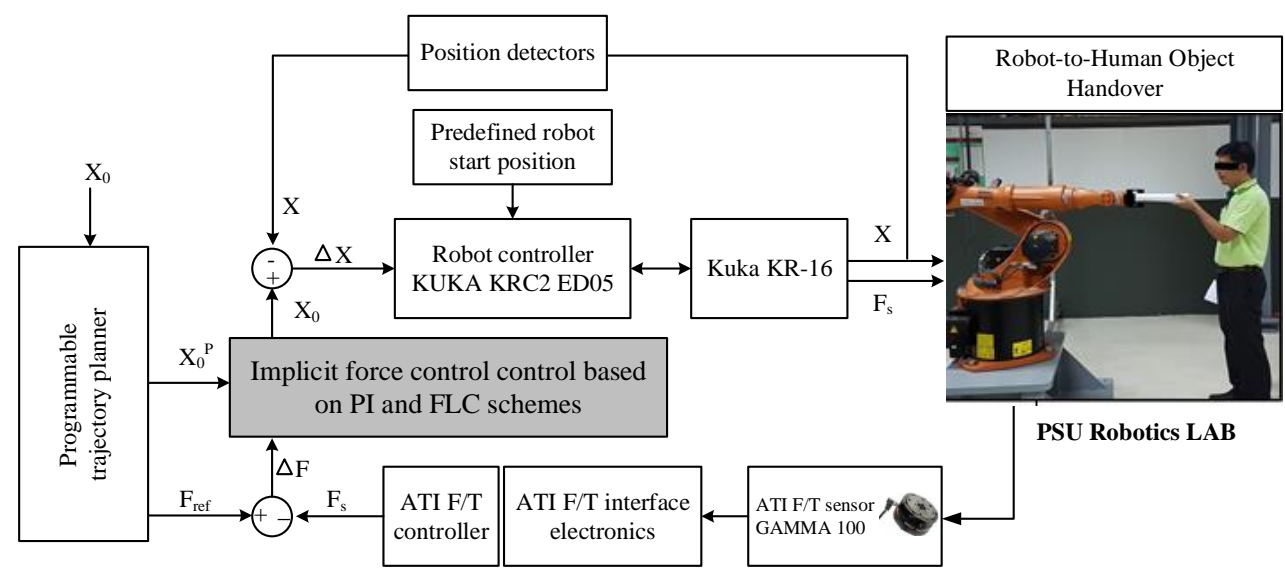

Figure 3. The Overall control schematic diagram developed for HRI object handover

\subsection{Evaluation of Robot Real-Time Path Control using Implicit Force Control}

It was necessary to evaluate the robot's real-time path control system to ensure effective HRI performance, thus a set of preliminary experiments were carried out. The main objective was to assess the performance of the robot real-time path control in terms of its reliability and accuracy. The robot was required to move along circular paths of 100, 150 and $200 \mathrm{~mm}$ in diameter in a fixed time period, whereas its motion was simulated and drawn using 1500 points $(\mathrm{N})$ in which the step size is defined by $2 \pi / \mathrm{N}$. To evaluate the quantitative performance of the control function, the robot's actual positions were compared to the desired values, and the following data recorded and compared, namely demanded, received (through TCP/IP) and actual values. Demanded values of incremental position were used to modify the robot's path and were generated in the external real-time Linux PC using MATLAB and directly transmitted to the KUKA controller via an Ethernet port using the TCP/IP protocol with a $4 \mathrm{~ms}$ cycle interrupt. Subsequently, received data, which represent the information acquired by the controller, were computed to establish a 3 axis transformation matrix in order to enable the changes in actual robotic movements.

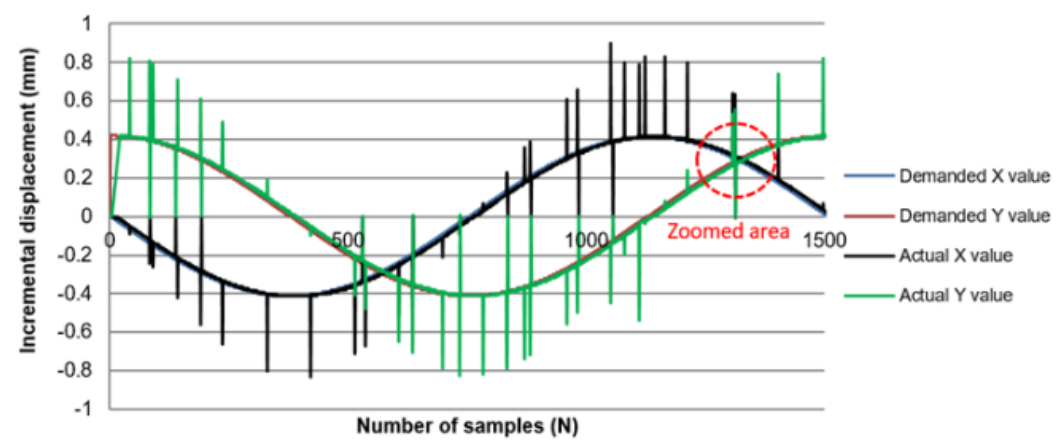

Figure 4. Results for a 100mm-diameter circular path using KUKA real-time path control (comparison of demanded and actual values)

It is evident that the desired values transmitted to the robot controller via the TCP/IP were fully achieved with a delay of $4 \mathrm{~ms}$. The time lag of $4 \mathrm{~ms}$ was attributed to the data transfer time between the RT Linux and the robot controller using TCP/IP communication. The experimental results show the presence of spikes in the profiles of the $x$ and $y$ coordinates of the robot, as shown in Figures 4 and 5 (representing the zoomed area). While the desired $\mathrm{x}$ and $\mathrm{y}$ path values received through TCP/IP communication were smooth, the actual $\mathrm{x}$ and $\mathrm{y}$ values showed pronounced spikes on the trajectories, but for only 1 sample period. These have been attributed to momentary errors occurring during executing of the real-time control command to initiate the incremental transition from one location to the next, largely as a result of attempting to run at 4 ms update rate. It can be said that the performance of the robotic control deteriorated at higher velocities of greater than $50 \mathrm{~mm} / \mathrm{s}$, where recorded position errors indicated larger variations. 
A careful observation of the system revealed that any attempt to further increase the sampling rate of the real-time path control system does not lead to an enhanced performance; however, this resulted in a much higher frequency of these errors, and therefore in this application, it was decided to maintain the data sampling rate at $4 \mathrm{~ms}$.

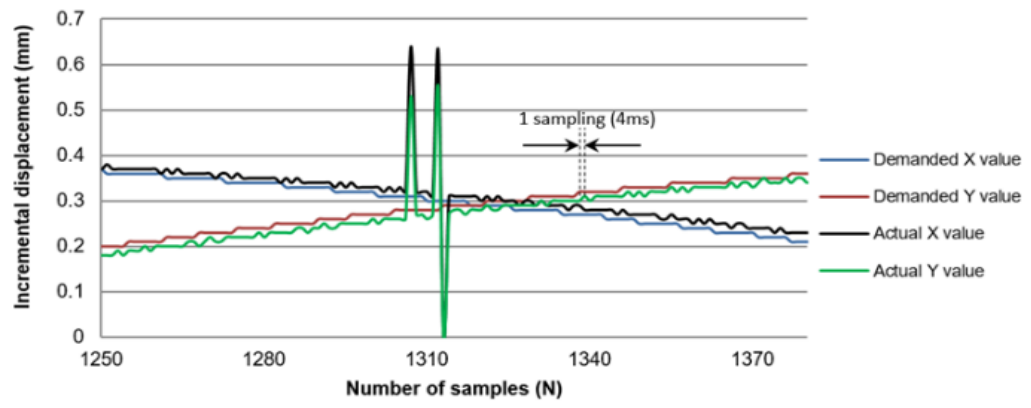

Figure 5. Results for a 100mm-diameter circular path using KUKA real-time path control (comparison of demanded and actual values)

The overall results indicating the overall average and corresponding standard deviations of the $x-y$ position errors whilst drawing the circular paths 100, 150 and $200 \mathrm{~mm}$ in diameter are summarized in Table 2 and Figure 6. The mean errors of the $\mathrm{x}$ and $\mathrm{y}$ axes slightly increased and varied from a minimum of $0.46 \%$ and $1.57 \%$ up to a maximum of $2.82 \%$ and $2.64 \%$ respectively. Additionally, the standard deviations of the two dimensions were in the range of $0.35-0.53 \mathrm{~mm}$. According to the results, it can be concluded that the performance of KUKA real-time control in path modification can be acceptable for the robot object-tohuman object handover; however, if you want to improve the path control performance, it can be achieved by decreasing the robot's end-effector velocity.

Table 2. Means and Standard Deviations Percentage Error for $\mathrm{x}-\mathrm{y}$ axes

\begin{tabular}{ccccccc}
\hline Test & \multicolumn{2}{c}{ Circle of 50mm diameter } & \multicolumn{2}{c}{ Circle of 75mm diameter } & \multicolumn{2}{c}{ Circle of 100mm diameter } \\
& Mean $(\%)$ & SD $(\%)$ & Mean $(\%)$ & SD $(\%)$ & Mean $(\%)$ & SD $(\%)$ \\
\hline x-axis & 0.46 & 0.44 & 0.84 & 0.43 & 2.82 & 0.39 \\
y-axis & 1.57 & 0.47 & 1.70 & 0.35 & 2.95 & 0.53 \\
\hline
\end{tabular}

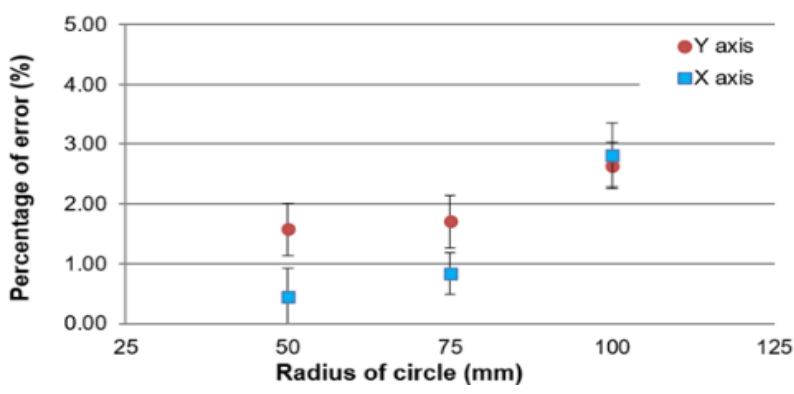

Figure 6. Means and standard deviations of the percentage of error for $x-y$ axes, while drawing circular paths 100,150 and $200 \mathrm{~mm}$ in diameter respectively

\subsection{Implementation of PI and FLC Schemes to Implicit Force Control}

After reviewed, researchers suggested that the position-based force control is easily implemented on various types of commercial robot controllers and it is also able to be appropriately associated with simple control schemes such as robust PI, PID or FLC algorithms. Therefore, in this study, it was decided to initially apply implicit force control based on PI control to provide the smallest possible error. The PI controller is preferable to PID control since the derivative term is more sensitive to noise, and this could introduce a destabilizing effect on the robot manipulator. However, the optimized PI controller has some disadvantages 
when it deals with non-linear behavioural problems such as high starting overshoot, sensitivity to controller gains and steady-state error. According to the complicated dynamic nature of human behaviour, FLC, which has a higher capability in dealing with non-linear dynamic responses, was, therefore, adopted [18-19]. A comparison of overall HRI qualitative performance of the control schemes was investigated. Figure 7 illustrates how implicit force control based on PI and FLC schemes can be developed and implemented to a robotic behaviour-based approach for safe physical HRI object handover.

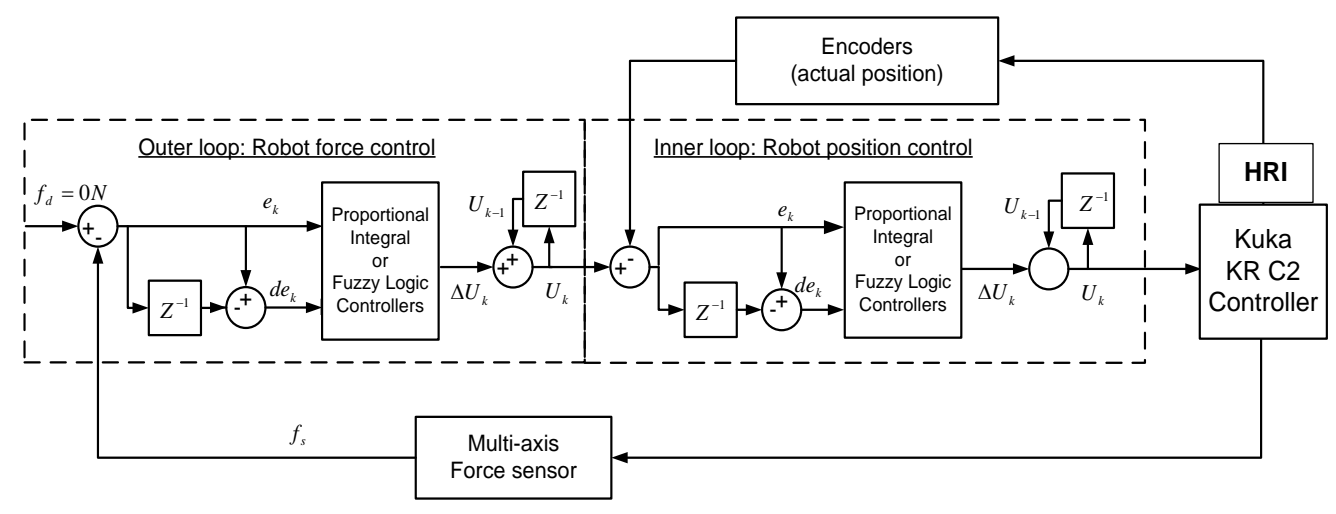

Figure 7. Schematic diagram of force control based on the PI or FLC controllers

Ziegler-Nichols method was used to determine the optimized PI force control's gain parameters, as it strives to keep the system overshoot response to a minimum and at the same time to provide rapid response. To confirm the appropriateness of the optimized gains, fast Fourier transform (FFT) was then applied to indicate the smallest oscillation moderating in the interactive force signal. As proposed by Neranon [12], a set of experiments was carried out to identify Kp and Tu parameters, and using ZieglerNichols technique appropriately provided the proportional gain $(\mathrm{Kp})$ of 0.35 and integral gain $(\mathrm{Ki})$ of 0.05 for the inner-loop position control, and the gain $\mathrm{Kp}$ of 0.35 and gain $\mathrm{Ki}$ of 0.05 were suitably achieved for the outer-loop force control scheme. Once the PI gains have been strategically achieved, subsequently the design of the FLC system is taken into consideration. This section presents the implementation of the implicit force control approach based on FLC for object handover. This control algorithm introduced by Zadeh is normally used to control non-linear or complex systems using 'IF-THEN' rules. The fuzzy inference emulates decision-making by applying the rule base, and the defuzzification process is applied to convert the results into a fuzzy output. The crisp sets of the fuzzy inputs and outputs have to be converted into linguistic forms using fuzzy membership functions [20]. In this study, the input and output variables of force and position control were normalized into five and seven linguistic levels respectively. The fuzzy rule-based systems are strategically developed and presented in Tables 3(a) and 3(b).

Table 3. Fuzzy rule Bases Designed for the Implicit Force Control Approach

\begin{tabular}{ccccccccccc}
\hline \multicolumn{4}{c}{ (a) Outer robot force contro } & \multicolumn{4}{c}{ Inner robot position control } \\
de & NL & NS & ZE & PS & PL & NL & NS & ZE & PS & PL \\
\hline NL & NL & NL & ZE & PS & PM & NL & NL & NS & NS & ZE \\
NS & NL & NL & ZE & PM & PL & NL & NM & ZE & ZE & PS \\
ZE & NL & NL & ZE & PL & PL & NM & NS & ZE & PS & PM \\
PS & NL & NM & ZE & PL & PL & NS & ZE & ZE & PM & PL \\
PL & NM & NS & ZE & PL & PL & ZE & PS & PS & PL & PL \\
\hline
\end{tabular}

Table 4. Comparisons of the Average Values and Standard Deviations of $f_{\max }, T_{t r f}$ and $W$

\begin{tabular}{|c|c|c|c|c|c|c|c|c|c|}
\hline \multirow{3}{*}{$\begin{array}{c}\text { Transfer velocity } \\
(\mathrm{mm} / \mathrm{s})\end{array}$} & \multirow{2}{*}{\multicolumn{3}{|c|}{$\begin{array}{l}\text { Human-human object handover } \\
\text { task }\end{array}$}} & \multicolumn{6}{|c|}{ Robot-to-human handover task } \\
\hline & & & & \multicolumn{3}{|c|}{ Robot PI force control } & \multicolumn{3}{|c|}{ Robot FLC force control } \\
\hline & $\begin{array}{l}\text { Work done/std } \\
(\mathrm{mJ})\end{array}$ & $\begin{array}{l}\text { Time/std } \\
\text { (s) }\end{array}$ & $\begin{array}{l}\text { Force/std } \\
(\mathrm{N})\end{array}$ & $\begin{array}{l}\text { Work done/std } \\
(\mathrm{mJ})\end{array}$ & $\begin{array}{l}\text { Time/std } \\
\text { (s) }\end{array}$ & $\begin{array}{l}\text { Force/std } \\
(\mathrm{N})\end{array}$ & $\begin{array}{l}\text { Work done/std } \\
(\mathrm{mJ})\end{array}$ & $\begin{array}{l}\text { Time/std } \\
\text { (s) }\end{array}$ & $\begin{array}{c}\text { Force/std } \\
(\mathrm{N})\end{array}$ \\
\hline 10 & $2.5 / 0.3$ & $0.6 / 0.06$ & $1.0 / 0.16$ & $4.6 / 0.8$ & $0.7 / 0.05$ & & & $0.6 / 0.07$ & $1.2 / 0.13$ \\
\hline 50 & & & & & & & & & \\
\hline 100 & $44.5 / 6.6$ & $0.4 / 0.03$ & $2.9 / 0.59$ & $150.8 / 9.9$ & $0.5 / 0.06$ & $5.4 / 0.54$ & $107.2 / 7.5$ & $0.5 / 0.05$ & $4.4 / 0.45$ \\
\hline
\end{tabular}




\section{SAFETY ISSUE IN ROBOT-TO-HUMAN OBJECT HANDOVER}

Safety is a key issue in HRI and one of the principal challenges in the development of the HRI object handover task because any failures which occur might become very critical. Hence, this section proposes the hardware and software safety issues in HRI, which have been given due consideration [21]. Firstly, a safeguarded zone [22] was introduced to conduct a safety strategy in the HRI test. If an unauthorised human enters the safeguarded zone, then the robot will initiate an immediate emergency stop. The robot working area was also optimized to minimize the risk of an injury. Stand-alone and manual control panel (MCP) emergency stop buttons were used to be manually activated by the human operator when accidents are detected. Additionally, the speed of the robot end effector allowed in the test was limited to $150 \mathrm{~mm} / \mathrm{s}$. Another key safety feature was developed based on control software, which is made up of a timeout assigned for the serial and TCP/IP communication. The waits for data transfer was implemented; if the transmission has not been completed yet after the timeout has elapsed, then all communication will be immediately terminated. Monitoring the interactive force feedback signal between the robot and the human was also established. Three safety thresholds for all handling interactive force signals in three dimensions were individually defined and If one or more force data (fx, fy and fz) are greater than the threshold (fthr) values, then the robot will be suddenly operated to release the object in order to ensure the safety of the human participant, and all robot functions will be also terminated.

\section{EVALUATION OF IMPLICIT FORCE CONTROL FOR ROBOT-TO-HUMAN OBJECT HANDOVER}

This section presents the evaluation of the performance of the HRI object handover tasks in order to conduct a quantitative assessment of the system's performance compared to that of the HHI handover. Moreover, a questionnaire technique was used in order to gather information from the selected participants concerning their evaluations of the robot control system. The test procedure for robot-to-human object handover permits the robot as a giver to transfer the object to the human receiver under the proposed conditions. Likewise, the HHI analysis, the interactive task is categorized into three phases: sending, transferring and receiving phases. Changing the different required conditions in the handover task led to corresponding changes in fmax, Ttrf and W. The same group of the selected participants were required to participate in the HRI tests.

\subsection{Test Results and Discussion of Robot-to-Human Object Handover}

A set of the HRI tests was undertaken to provide a comparison of the performance of the robot to human behaviour. Owing to the investigation of the HHI results, the graph trends of interactive force profiles carried out from all desired scenarios are quite similar. Contrastly, the fmax and $\mathrm{W}$ parameters achieved from $\mathrm{HHI}$ are proportional to masses added to the baton-type object whereas the term of Ttrf is slightly inversely proportional to the added mass. To avoid further complicated analysis in HRI, hence, the total object mass of $0.42 \mathrm{~kg}$ only was specifically tested at the demanded velocities of 10,50 and $100 \mathrm{~mm} / \mathrm{s}$, so that a greater variety of object loads can be ignored. According to the HRI experimental outcomes, it presents the timedomain profiles of the interactive force between the robot and human-based on PI and FLC implicit force control. Again, the process of graph normalization and curve fitting were implemented, and $3 \sigma$ was statistically computed. MATLAB was used to determine the test results summarized in Table 4. The evaluation of the robot-to-human object handover can be addressed as the transfer times (Ttrf) are reduced with an increase in velocity for all scenarios, with the transfer time in the HHI tasks the fastest compared to those of PI and FLC. However, the transfer times with FLC are slightly quicker than those of with PI. According to the average fmax, these values vary with object transfer velocity. Generally, the interactive force increases with increasing demanded velocity, whereas the magnitudes of force in the HHI tests are again the smallest values for all cases, followed by HRI with FLC and then HRI with PI. The work done $(\mathrm{W})$ can be observed that the trends for $\mathrm{W}$ and fmax are quite similar, where the amount of work done significantly increase with higher demanded velocity. Again, it can be concluded that the test performance achieved by HHI tasks is better than that of HRI with either PI or FLC, and furthermore, the work done in HRI with FLC is also slightly lower than that of PI control.

\subsection{Frequency Domain Analysis of Human-Robot Handover Task}

Frequency domain analysis, by way of the fast Fourier transform (FFT), was used to identify the performance of the robot force and velocity control in the HRI tasks. The FFT analysis technique has been applied in many engineering applications [23]. In this study, FFT was employed to determine changes in the power spectrum of the HRI system, and when the robot manipulator was controlled using PI and FLC force

Implicit force control approach for Safe physical robot-to-human object handover (Paramin Neranon) 
and velocity control schemes. From the experimental results showing the FFT analysis results of the oscillation moderating in the interactive force signals. An FFT was applied to extract the noise frequencies, which were used to compare and evaluate the qualitative performance of the robot based on the PI and FLC schemes at various object transfer speeds. The following examples show how the performance in terms of the robot force and velocity control can be evaluated using FFT analysis where the robot passed the object to the human at

a velocity of $100 \mathrm{~mm} / \mathrm{s}$. Figures 8 (a and c) illustrate the actual interactive force trajectories during the transfer phase using PI and FLC force control. To appropriately identify the noise signal, a high-pass filter (HPF) with a cut-off frequency at $10 \mathrm{~Hz}$ was used to filter the data. The results of the FFT analysis are plotted in Figures

8 (b and d), where the maximum power spectral density of PI control is approximately $49.8 \mathrm{~N} 2$ at a frequency of $28.66 \mathrm{~Hz}$. When the robot was controlled using FLC, a corresponding set of results can be seen to reduce the magnitudes of the filtered noise signal in the system. Also, the maximum peak of the power spectrum is reduced to $20.81 \mathrm{~N} 2$ at the frequency of $26.52 \mathrm{~Hz}$.

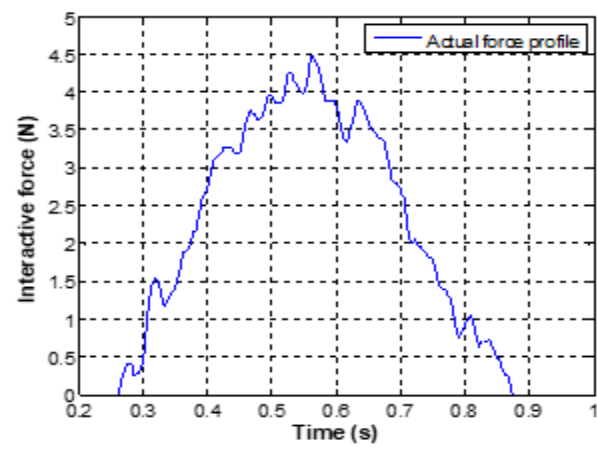

(a) Actual force profile (PI)

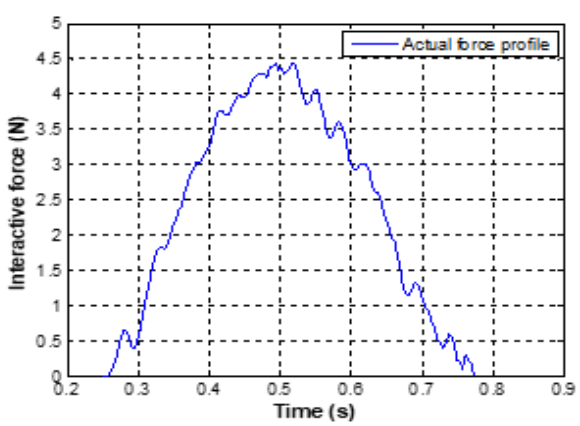

(c) Actual force profile (FLC)

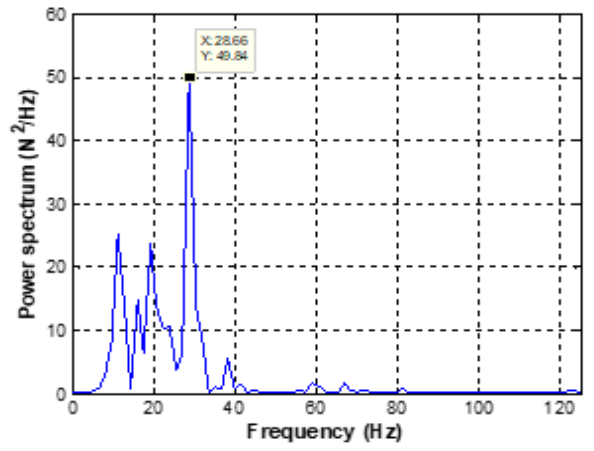

(b) Power spectrum (PI)

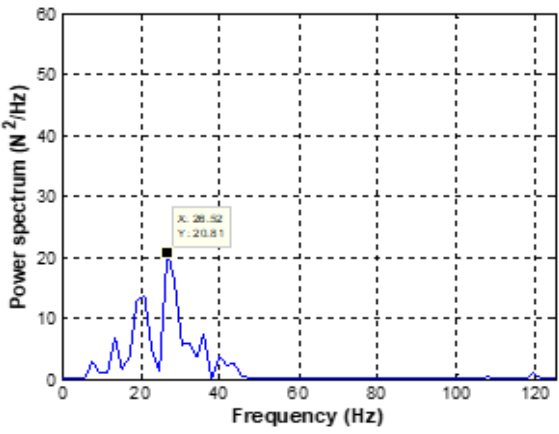

(d) Power spectrum (FLC)

Figure 8. Actual force profiles and FFT analysis for HRI using PI (a-b) and FLC (c-d) control

The evaluation of the robot performance for all demanded velocity conditions in the robot-to-human handover tasks based on PI and FLC algorithms is summarised in Table 5. The results are expressed as the frequencies of the maximum power spectral densities delivered according to the proposed conditions, which varied slightly in the range of approximately $15-34 \mathrm{~Hz}$. These dominant frequencies were conducted from the dynamics of the robot force control system. According to the results, the magnitudes of the maximum power spectrum of the filtered data were examined to determine the oscillations of the noise signal for each scenario. FLC provided a better qualitative performance of the robot in all robot-to-human handover tasks. A comparison of the power spectral densities using FLC and PI control shows that the values of the power spectrum were respectively 5.4, 24.3 and 28.6N2 for FLC, and 7.7, 34.7 and 42.5N2 for PI control. It can be concluded that the fuzzy logic force control method is more effective in giving enhanced stability and greater overall qualitative performance in the system. 
Table 5. Qualitative Performance Measurement from FFT Analysis in the Robot-Human Handover Tasks Represented by the Maximum Power Spectral Values and Their Frequencies

\begin{tabular}{ccccccc}
\hline \multirow{2}{*}{ Velocity $(\mathrm{mm} / \mathrm{s})$} & \multicolumn{3}{c}{ HRI using PI } & \multicolumn{3}{c}{ HRI using FLC } \\
& Mean $\left(\mathrm{N}^{2}\right)$ & $\mathrm{SD}\left(\mathrm{N}^{2}\right)$ & Frequency $(\mathrm{Hz})$ & Mean $\left(\mathrm{N}^{2}\right)$ & $\mathrm{SD}\left(\mathrm{N}^{2}\right)$ & Frequency $(\mathrm{Hz})$ \\
\hline 10 & 7.7 & 3.3 & $15.5-30.1$ & 5.4 & 3.1 & $17.6-33.2$ \\
50 & 34.7 & 12.6 & $17.5-28.6$ & 24.3 & 7.4 & $18.2-27.3$ \\
100 & 42.5 & 14.3 & $16.7-32.1$ & 28.6 & 9.0 & $18.7-31.3$ \\
\hline
\end{tabular}

\subsection{Analysis of Questionnaire Responses of Robot-to-Human Object Handover}

A questionnaire was designed based on the suggestions in a standard textbook of survey research [24] and also used in order to gather information from the subjects concerning their evaluations of the robot control system in the object handover test. The rating scale technique was applied to convey how comfortable the participants were with the robot's performance in the HRI handover tasks and how close the qualitative performance of the HRI is to that of HHI object handover. Furthermore, as suggested by Bellera et al. [25], the paired t-test comparison technique, which is typically used to compare the means of two different groups on different occasions with repeated measurements and the same samples, was statistically implemented. The survey responses based on the rating scale items for the evaluation of the robot control system in HRI are shown in Table 6.

Table 6. Survey Responses for the Robot-To-Human Object Handover Tasks

\begin{tabular}{|c|c|c|c|c|c|c|}
\hline No & Question & $\begin{array}{c}\text { Poor } \\
(1)\end{array}$ & $\begin{array}{c}\text { Fair } \\
(2)\end{array}$ & $\begin{array}{l}\text { Good } \\
\text { (3) }\end{array}$ & $\begin{array}{c}\text { Very } \\
\text { Good (4) }\end{array}$ & $\begin{array}{l}\text { Excellent } \\
\quad(5)\end{array}$ \\
\hline 1 & $\begin{array}{l}\text { How comfortable were you with the robot- to-human object handover } \\
\text { based on PI? }\end{array}$ & - & 2 & 3 & 13 & 0 \\
\hline 2 & $\begin{array}{l}\text { How comfortable were you with the robot-to-human object handover } \\
\text { based on FLC? }\end{array}$ & - & 1 & 1 & 3 & 13 \\
\hline 3 & $\begin{array}{l}\text { Rate the stability/reliability of the overall performance of HRI tests } \\
\text { using PI? }\end{array}$ & - & 2 & 4 & 10 & 2 \\
\hline 4 & $\begin{array}{l}\text { Rate the stability/reliability of the overall performance of HRI tests } \\
\text { using FLC? }\end{array}$ & - & 1 & 1 & 9 & 7 \\
\hline 5 & $\begin{array}{l}\text { How do you compare the HRI object handover to the HHI object } \\
\text { handover task? }\end{array}$ & - & - & 2 & 13 & 3 \\
\hline
\end{tabular}

\subsubsection{How Comfortable Were you with the Robot HRI using PI and FLC}

The statistical analysis by means of the Wilcoxon signed-rank test was conducted using the statistical package for the social sciences (SPSS), which is one of the most powerful programs used to analyze a wide variety of statistical problems. A confidence interval of $95 \%$ or a significance level $(\alpha)$ of 0.05 , which is typically accepted and recommended for scientific experiments, was adopted in these statistical tests. This section evaluates how comfortable the participants felt in participating in the robot-tohuman handover tasks with the KUKA robot manipulator based on the PI and FLC schemes. The hypothesis $H_{0}$ and alternative hypothesis $H_{l}$ for the Wilcoxon signed-rank two-tailed test [26] are given below:

$H_{0}$ : The median difference between pairs of observations is zero so that there is no significant difference in being comfortable with the robot controlled by the PI and FLC schemes.

$H_{1}$ : The median difference between pairs of observations is not equal to zero so that there is a significant difference in being comfortable with the robot controlled by the PI and FLC.

The results of how the eighteen participants were comfortable with RHI (1-2) show that two largest groups of 13 participants each, or $72.2 \%$, were either satisfied or very satisfied with the performance of the robot implemented by PI and FLC force control respectively. The t-test analysis results in Table 7 show the average satisfaction ratings based on PI and FLC are 3.61 and 4.56, with the corresponding standard deviations of 0.69 and 0.85 respectively. The rank table provides information on the comparison of both control methods. The negative ranks present that only one participant preferred the PI force control scheme to FLC. In contrast, the positive ranks indicate that 15 people thought that the qualitative performance of FLC was significantly better than PI control. The number of ties demonstrates two participants who rated for both robot force control schemes equally. The negative mean rank value of 7.50 is less than the positive mean rank of 8.57. Moreover, the test statistics identify the overall significance of the difference between the two control methods. In other words, the human participants were more comfortable in participating with the robot using the FLC force control rather than PI control. Additionally, the test statistics identify the overall significance of the difference between the two control methods. The significance value estimated was calculated as 0.001 , which is less than 0.05 , and therefore it can be concluded that the hypothesis $H_{0}$ has to be clearly rejected, and the alternative hypothesis $H_{l}$ has to be accepted. In other words, there is a significant 
difference in the population distributions from the samples derived, and the human participants were more comfortable in participating with the robot using the FLC rather than PI control.

Table 7. Statistical T-test Results

\begin{tabular}{cccccc}
\hline Descriptive Statistics & $\mathrm{N}$ & Mean & Std. Deviation & Minimum & Maximum \\
\hline PI and FLC & 18 & 3.61 & 0.69 & 2 & 4 \\
for RHI & 18 & 4.56 & 0.85 & 2 & 5 \\
\hline \multicolumn{7}{c}{ Ranks } & & & & \\
\hline & & Negative Ranks & $1^{\text {a }}$ & Mean Rank & Sum of Ranks \\
\hline FLC and PI for RHI & Positive Ranks & $15^{\text {b }}$ & 8.57 & 7.50 \\
& Ties & $2^{\text {c }}$ & & 128.50 \\
& Total & 18 & & \\
\hline a. FLC for RHI < PI for RHI, b. FLC for RHI > PI for RHI and c. FLC for RHI = PI for RHI
\end{tabular}

\begin{tabular}{cc}
\hline Test Statistics $^{\mathbf{a}}$ & FLC and PI for RHI \\
\hline $\mathrm{Z}$ & $-3.397^{\mathrm{b}}$ \\
Asymp. Sig. (2-tailed) & .001 \\
\hline a. Wilcoxon Signed-Ranks Test and b. Based on negative ranks.
\end{tabular}

\subsubsection{Rate the Stability/Reliability of the PI and FLC HRI Tests}

The same statistical analysis technique was also implemented to assess the responses of the participants comparing the overall stability of the robot control schemes. The paired T-test results are expressed in Table 8. According to the stability and reliability of the robot's performance (3-4), the t-test results reveal that ratings of the stability of the robot system based on the PI and FLC were 3.67 (Std. of 0.84) and 4.22(Std. of 0.81 ) respectively. The results of the statistical analysis show as follows: $5.5 \%$ of samples preferred the stability of PI to FLC, 55.5\% of participants were more appreciative of the stability performance of FLC rather than PI control, and 39\% perceived similar levels of stability for both robotic control techniques. The value of the positive mean rank (FLC) was 6.05, which is higher than the negative mean rank value (PI) at 5.50, which suggests that the participants preferred the stability of the robot performance based on FLC to that of PI. The overall significance value of the Wilcoxon signed-rank twotailed test was 0.008 , and it can be concluded that there is a significant difference in the population distributions from the participants who were more comfortable with the robot's stability using fuzzy logic control rather than the proportional integral control scheme in the HRI tasks.

Table 8. Statistical T-test Results

\begin{tabular}{cccccc}
\hline Descriptive Statistics & N & Mean & Std. Deviation & Minimum & Maximum \\
\hline Stability_of_PI & 18 & 3.67 & 0.84 & 2 & 5 \\
Stability_of_FLC & 18 & 4.22 & 0.81 & 2 & 5 \\
\hline \multicolumn{2}{c}{ Ranks } & & $\mathrm{N}$ & Mean Rank & Sum of Ranks \\
\hline & Negative Ranks & $1^{\mathrm{a}}$ & 5.50 & 5.50 \\
Stability of FLC and PI & Positive Ranks & $10^{\mathrm{b}}$ & 6.05 & 60.50 \\
& Ties & $7^{\mathrm{c}}$ & & \\
& Total & 18 & & \\
\hline a. Stability of FLC < Stability of PI, b. Stability of FLC > Stability of PI and c. Stability of FLC = Stability of PI
\end{tabular}

\begin{tabular}{lc}
\hline Test Statistics $^{\text {a }}$ & Stability_of_FLC and Stability_of_PI \\
\hline Z & $-2.673^{\mathrm{b}}$ \\
Asymp. Sig. (2-tailed) & .008 \\
\hline a. Wilcoxon Signed Ranks Test \\
b. Based on negative ranks.
\end{tabular}

\subsubsection{Comparison HRI to HHI Object Handover Tasks}

Finally, a formal overall comparison between HRI handover and HHI handover was undertaken, and this shows that, in brief, the majority of the participants at 13 of the sample (72\%) were satisfied with the robot's performance in HRI, while 2 people (11\%) were neither satisfied nor dissatisfied. On the other hand, 3 participants (17\%) were very satisfied with the effective implementation of the HRI object handover tests. Based on a five-point rating, an average of 4.06 was obtained for satisfaction with the robot implementation 
of the HRI task as compared with the HHI task. Therefore, it can be summarised that both PI and FLC robot control strategies provide effective performance and reliability of the robot-to-human object handover, where the robot was able to successfully transfer the baton object to the human in a smooth, safe and reliable manner. However, careful observation of the interactive force profiles for FLC revealed that these profiles are moderately smoother than the PI implementation. Furthermore, FLC was shown to provide improved performance of the robot force control system over that of PI force control, in terms of reduced interactive force, shorter transfer time and lower work done. This also supports the conclusion that using FLC has a significant advantage when controlling non-linear systems [27], and is more insensitive than PI control to variations in small external force disturbances, which is an important requirement in robot force/position control schemes [28].

\section{CONCLUSION}

This research focuses on the development of appropriate human-like robot control able to effectively perform human-like functions in the safe HRI object handover tasks. The behavioural control strategies developed by first understanding how an equivalent HHI object handover can be used to establish a framework for a robotic behaviour-based approach. The results of interactive experiments with robot-tohuman handover tasks allowed a comparison of the implicit position-based force control using PI and FLC algorithms. It can be concluded that the quantitative performance of the PI and FLC force control schemes is considered acceptable for the physical object handover tasks. Nevertheless, after careful observation with regard to the HRI handover test results, FLC was shown to be slightly superior to PI control by actively compensating for the dynamics in the non-linear system and demonstrated better overall performance and stability. Moreover, the results of survey responses from the participants were in agreement with the parallel test outcomes, demonstrating significant satisfaction with the overall performance of the HRI system, as measured by an average rating of 4.06 on a five-point scale. In brief, this research has contributed the foundations for long-term research, particularly in the development of an interactive real-time robot-force control system, which enables the robot manipulator arm to cooperate with a human to facilitate the dextrous transfer of objects in a safe and speedy manner. It can be also said that the safety systems proposed are working effectively and thus avoiding the likelihood of unsafe HRI handover actions being taken.

\section{ACKNOWLEDGEMENTS}

I would like to express my sincere gratitude to all senior researchers, technicians and staff at the Department of Mechanical Engineering, Faculty of Engineering at Prince of Songkla University for all their help, support, encouragement, advice, motivation, enthusiasm, immense knowledge and guidance given throughout the haptic human-robot interaction research.

\section{REFERENCES}

[1] Jevtić A., Doisy G., Bodiroža S., Edan Y. and Hafner V. V., "Human-Robot Interaction through 3D Vision and Force Control", 9th ACM/IEEE International Conference on Human-Robot Interaction (HRI), Bielefeld, 2014, pp. 102-102.

[2] Bicchi A., "Of Robots, Humans, Bodies and Intelligence: Body Languages for Human-Robot Interaction", 10th ACM/IEEE International Conference on Human-Robot Interaction (HRI), Portland, OR, 2015, pp. 1-1.

[3] Parastegari S., Noohi E., Abbasi B. and Žefran M., "Failure Recovery in Robot-Human Object Handover", IEEE Transactions on Robotics, 2018, vol. 34, no. 3, pp. 660-673.

[4] Cakmak, M., Srinivasa, S. S., Min Kyung, L., Forlizzi, J. and Kiesler, S. "Human preferences for robot-human hand-over configurations", Intelligent Robots and Systems (IROS), 2011. pp. 1986-19931.

[5] Parastegari S., Abbasi B., Noohi E. and Zefran M., "Modeling human reaching phase in human-human object handover with application in robot-human handover", IEEE/RSJ International Conference on Intelligent Robots and Systems (IROS), Vancouver, BC, 2017, pp. 3597-3602.

[6] Obo T., Kawabata R. and Kubota N., "Cooperative Human-Robot Interaction Based on Pointing Gesture in Informationally Structured Space", World Automation Congress (WAC), Stevenson, WA, 2018, pp. 1-5.

[7] Strabala, K. W., Lee, M. K., Dragan, A. D., Forlizzi, J. L., Srinivasa, S., Cakmak, M. and Micelli, V. "Towards seamless human-robot handovers", Journal of Human-Robot Interaction, 2013, 2, (1), pp. 112-132.

[8] Wesley, P. C., Chris, A. C. P., Loos, H. F. M. V. d. and Elizabeth, A. C. (2012) "Grip forces and load forces in handovers: implications for designing human-robot handover controllers", Proceedings of the seventh annual ACM/IEEE international conference on Human-Robot Interaction. Boston, Massachusetts, USA, 2012, pp. 9-16.

[9] Mason, A. and MacKenzie, C. "Grip forces when passing an object to a partner", Experimental Brain Research, 2005, 163, pp. 173-187. 15p. doi:10.1007/s00221-004-2157. 
[10] Lee, M. K., Tang, K. P., Forlizzi, J. and Kiesler S., "Understanding users! Perception of privacy in human-robot interaction", 6th ACM/IEEE International Conference on Human-Robot Interaction (HRI), Lausanne, 2011, pp. 181-182.

[11] Reed, K. B. and Peshkin, M. A. "Physical Collaboration of Human-Human and Human-Robot Teams", IEEE Transactions on Haptics, 2008, 1, (2), pp. 108-120.

[12] Neranon, P. "Robot-to-Human Object Handover using a Behavioural Control Strategy", Proceedings of the 2018 IEEE 5th International Conference on Smart Instrumentation, Measurement and Applications (ICSIMA 2018), Songkhla, Thailand, 2018.

[13] De Schutter, J. and Van Brussel, H. "Compliant robot motion II. A control approach based on external control loops", Int. J. Rob. Res., 1988, 7, (4), pp. 18-33.

[14] Neranon P. and Bicker R., "Human-human interaction using a behavioural control strategy", 2013 IEEE Conference on Technologies for Practical Robot Applications (TePRA), Woburn, MA, 2013, pp. 1-6.

[15] Faruq, A., Nor Shah, M. F. and Abdullah, S. S. " Multi-objective Optimization of PID Controller using Paretobased Surrogate Modeling Algorithm for MIMO Evaporator System ", International Journal of Electrical and Computer Engineering, 2018, 8(1), pp. 556-565

[16] Nguyen H.Q., Nguye, P., Dao, P.N and Nguyen, T.B., "Multiparametric model predictive control based on laguerre model for permanent magnet linear synchronous motors", International Journal of Electrical and Computer Engineering (IJECE), 2019, ISSN: 2088-8708, vol.9, no.2, pp. 1067-1077.

[17] Farid, F. and Redouane, T. "Dynamic External Force Feedback Loop Control of a Robot Manipulator Using a Neural Compensator-Application to the Trajectory Following in an Unknown Environment", Int. J. Appl. Math. Comput. Sci., 2009, 19, (1), pp. 113-126.

[18] Khanke, P. K. and Jain, S. D., "Comparative analysis of speed control of BLDC motor using PI, simple FLC and Fuzzy - PI controller", 2015 International Conference on Energy Systems and Applications, Pune, 2015, pp. 296-301.

[19] Amuthameena S. and Monisa S., "Design of fuzzy logic controller for a non-linear system", IEEE International Conference on Electrical, Instrumentation and Communication Engineering (ICEICE 2017), Karur, 2017, pp. 1-7.

[20] Ra vi Sankar, R.S., Jayaram Kumar, S.V. and Mohan Rao, G. "Adaptive Fuzzy PI Current Control of Grid Interact PV Inverter", International Journal of Electrical and Computer Engineering (IJECE), 2018, ISSN: 2088-8708, vol.8, no.1, pp. 472-482.

[21] Murphy, R. R. and Schreckenghost, D., "Survey of metrics for human-robot interaction", 2013 8th ACM/IEEE International Conference on Human-Robot Interaction (HRI), Tokyo, 2013, pp. 197-198.

[22] Yamada, Y., Hirasawa, Y., Huang, S., Umetani, Y. and Suita, K. "Human-robot contact in the safeguarding space", Mechatronics, IEEE/ASME Transactions, 1997, 2, pp. 230-236.

[23] Susana, V. A., Joaquin, F. O. and Fredy, E. H. "Measurement of low frequency mechanical vibrations based on an inverted magnetic pendulum", International Journal of Electrical and Computer Engineering (IJECE), 2019, ISSN: 2088-8708, vol.9, no.5, pp. 3480-3487.

[24] Marsden, P. V. and Wright, J. D. "Handbook of survey research". Second Edition ed: Bingley, UK: Emerald Publishing Group Limited., 2010.

[25] Bellera, C. A., Julien, M. and Hanley, J. A. "Normal approximations to the distributions of the Wilcoxon statistics: accurate to what n? Graphical insights", Journal of Statistics Education, 2010, 18, pp. 1-17.

[26] Zhang, R., Li, Y. and Li, X., "Topology Inference With Network Tomography Based on t-Test", IEEE Communications Letters, 2014, vol. 18, no. 6, pp. 921-924.

[27] Mayub, A. and Fahmizal, "Center of Pressure Feedback for Controlling the Walking Stability Bipedal Robots using Fuzzy Logic Controller", International Journal of Electrical and Computer Engineering (IJECE), 2018, ISSN: 2088-8708, vol.8, no.5, pp. 3678-3696.

[28] Gaurav and Amrit, K. "Comparison between Conventional PID and Fuzzy Logic Controller for Liquid Flow Control: Performance Evaluation of Fuzzy Logic and PID Controller by Using MATLAB/Simulink", International Journal of Innovative Technology and Exploring Engineering, 2012, ISSN: 2278-3075, vol.1, no.1.

\section{BIOGRAPHIES OF AUTHORS}

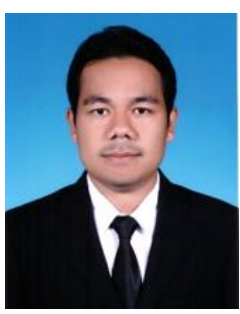

Paramin Neranon received a B.E. degree in Mechatronics Engineering and M.Eng degree in Electrical Engineering from Prince of Songkla University, Thailand, in 2003. I achieved a Ph.D. degree in Mechanical and systems engineering at Newcastle University, UK, in 2015. In the same year (2015) I have been with the Department of Mechanical Engineering at Prince of Songkla University, as a Mechatronics lecturer. My current research interests include HumanRobot Interaction, Industrial and mobile robotics, Robot (position/force/velocity) control, Embedded sensing and measurement systems, Multi-axis motion control systems, Real-time control, Mechatronic system, Neural Network \& Fuzzy Logic Control, Condition Monitoring, Modelling and Simulation, Rehabilitation and Medical Robotics etc. 